\title{
A MASSIVE PROGENITOR OF THE LUMINOUS TYPE IIn SUPERNOVA 2010j1
}

\author{
Nathan Smith ${ }^{1}$, Weidong Li ${ }^{2}$, Adam A. Miller ${ }^{2}$, Jeffrey M. Silverman ${ }^{2}$, Alexei V. Filippenko $^{2}$, \\ Jean-Charles Cuillandre ${ }^{3}$, Michael C. Cooper ${ }^{4}$, Thomas Matheson ${ }^{5}$, and Schuyler D. Van DyK ${ }^{6}$ \\ ${ }^{1}$ Steward Observatory, University of Arizona, 933 North Cherry Avenue, Tucson, AZ 85721, USA; nathans @ as.arizona.edu \\ ${ }^{2}$ Department of Astronomy, University of California, Berkeley, CA 94720-3411, USA \\ ${ }^{3}$ Canada-France-Hawaii Telescope Corporation, 65-1238 Mamalahoa Hwy., Kamuela, HI 96743, USA \\ ${ }^{4}$ Department of Physics and Astronomy, University of California, 4129 Frederick Reines Hall, Irvine, CA 92697-4575, USA \\ ${ }^{5}$ National Optical Astronomy Observatory, 950 North Cherry Avenue, Tucson, AZ 85719-4933, USA \\ ${ }^{6}$ Spitzer Science Center, California Institute of Technology, Mail Code 220-6, 1200 East California Boulevard, Pasadena, CA 91125, USA \\ Received 2010 November 17; accepted 2011 March 1; published 2011 April 15
}

\begin{abstract}
The bright, nearby, recently discovered supernova (SN) 2010jl is a luminous Type IIn SN. Here, we report archival Hubble Space Telescope (HST) observations of its host galaxy UGC 5189A taken roughly $10 \mathrm{yr}$ prior to explosion, as well as early-time optical spectra of the SN. The HST images reveal a luminous, blue point source at the position of the SN, with an absolute magnitude of -12.0 in the F300W filter. If it is not just a chance alignment, the source at the SN position could be (1) a massive young ( $<6 \mathrm{Myr}$ ) star cluster in which the SN resided, (2) a quiescent, luminous blue star with an apparent temperature around 14,000 K, (3) a star caught during a bright outburst akin to those of luminous blue variables, or (4) a combination of option (1) and option (2) or (3). Although we cannot confidently choose between these possibilities with the present data, any of them imply that the progenitor of SN $2010 \mathrm{jl}$ had an initial mass above $30 M_{\odot}$. This reinforces mounting evidence that many SNe IIn result from very massive stars, that massive stars can produce visible SNe without collapsing quietly to black holes, and that massive stars can sometimes retain their $\mathrm{H}$ envelopes until shortly before explosion. Standard stellar evolution models fail to account for these observed properties.
\end{abstract}

Key words: circumstellar matter - stars: evolution - stars: mass-loss - stars: winds, outflows - supernovae: general

Online-only material: color figures

\section{INTRODUCTION}

Supernova (SN) 2010j1 was discovered on 2010 November 3.52 (UT dates are used throughout this paper) by Newton \& Puckett (2010). With a discovery magnitude of 13.5 (unfiltered), this is one of the brightest $\mathrm{SNe}$ in recent years. After one day it continued to brighten (12.9 mag on 2010 November 4.50), signaling that it was also caught early in its evolution. Moreover, its host galaxy UGC 5189A is located at a distance of almost $50 \mathrm{Mpc}$, suggesting that SN 2010jl is intrinsically luminous, with a peak absolute magnitude of about -20 . Earlytime spectra showed that it is a Type IIn SN (Benetti et al. 2010; see also Schlegel 1990; Filippenko 1997). SNe IIn constitute about 6\%-9\% of core-collapse SNe (Smith et al. 2011a; Li et al. 2011).

We analyzed pre-explosion archival images of the field of SN 2010jl obtained with the Hubble Space Telescope (HST), and we obtained ground-based post-explosion images of the SN that allow us to constrain its position. We find a blue source in the $H S T$ images that is coincident with the $\mathrm{SN}$ position to within $1 \sigma$ of our astrometric solution, suggesting that the source is likely to be either a detection of the blue progenitor star itself or the star cluster in which it resided (or both). As discussed below, this progenitor candidate has important implications for $\mathrm{SNe}$ IIn, as well as for the evolution and death of massive stars in general.

There have been two previous claimed detections of progenitors of SNe IIn, and both were luminous stars that reinforce a suspected link between SNe IIn and the class of massive unstable stars known as luminous blue variables (LBVs). One case is SN 2005gl, which was a moderately luminous SN IIn (Gal-Yam et al. 2007). Pre-explosion images showed a source at the SN position that faded below detection limits after the SN had faded (Gal-Yam \& Leonard 2009). Its high luminosity suggested that the progenitor was a massive LBV. The other example of a claimed detection of an SN IIn progenitor-SN $1961 \mathrm{~V}$ - has a more complicated history because it is much closer to us and more highly scrutinized. For decades SN 1961V was considered a prototype (although the most extreme case) of giant eruptions of LBVs, and an analog of the 19th century eruption of $\eta$ Carinae (Goodrich et al. 1989; Filippenko et al. 1995; Van Dyk 2005). However, two recent studies (Smith et al. 2011b; Kochanek et al. 2010) argue for different reasons that SN 1961 V was probably a true core-collapse SN IIn. Both studies point out that the pre-1961 photometry of this source's variability was a detection of a very luminous quiescent star, as well as a precursor LBV-like giant eruption in the few years before the supposed core collapse. While the exact explosion mechanism of SN $1961 \mathrm{~V}$ is still debated (e.g., Van Dyk \& Matheson 2011), the clear detection and post-outburst fading of its LBV progenitor is as reliable as the case for SN 2005gl.

This connection between SNe IIn and LBVs based on their progenitor stars supports an existing link based on the physics of SN IIn explosions-namely, accounting for highly luminous SNe IIn with a blast wave hitting a massive opaque shell (e.g., Smith \& McCray 2007; van Marle et al. 2010) requires strong eruptive mass loss in the years preceding core collapse, consistent with giant eruptions of LBVs (Smith et al. 2007, 2008, 2010; Gal-Yam et al. 2007; Gal-Yam \& Leonard 2009). Additional reasons to suspect a connection between LBVs and SNe IIn are reviewed elsewhere (Smith 2008).

In this paper, we present a possible third detection of an SN IIn progenitor. This adds to a number of claimed progenitor detections of other SN types, most of which are 

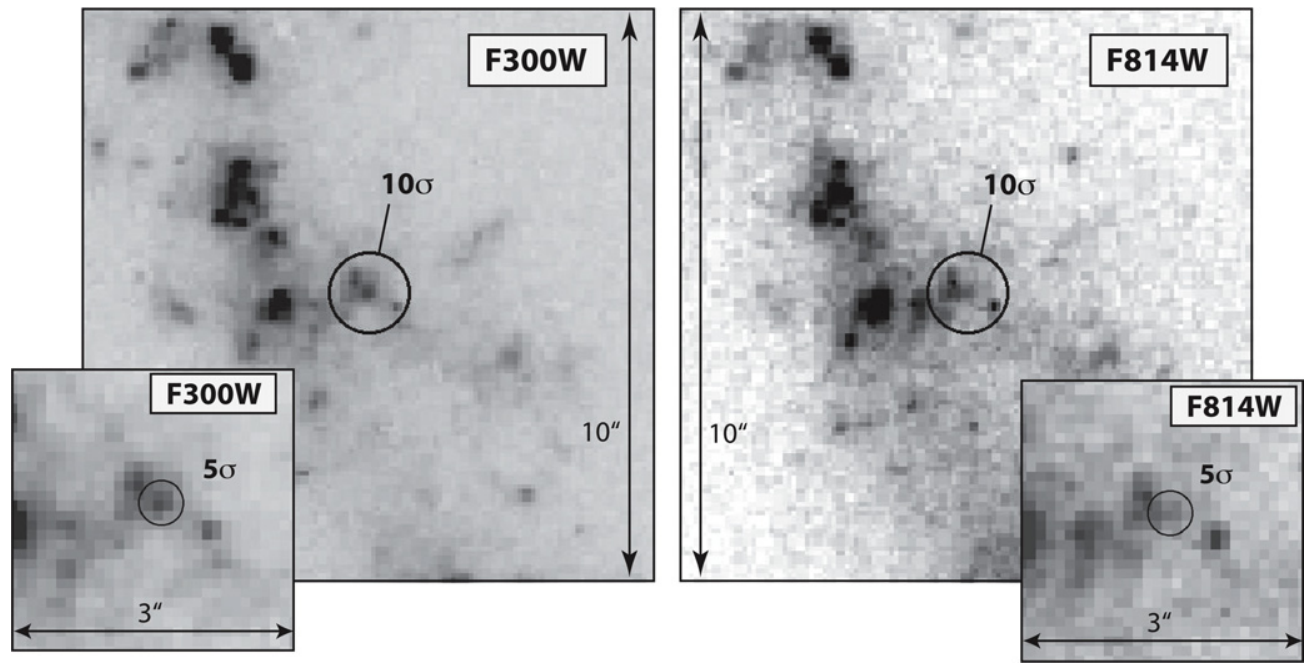

Figure 1. Images of the environment $\left(10^{\prime \prime} \times 10^{\prime \prime}\right)$ of SN 2010jl in its host galaxy, UGC 5189A. These are HST/WFPC2 images in the F300W and F814W filters, obtained in 2001 February. North is up and east is to the left. The circle has a radius of 0 ". 47, which is 10 times the $1 \sigma$ uncertainty of our astrometric solution. The smaller panels show zoomed-in $\left(3^{\prime \prime} \times 3^{\prime \prime}\right)$ regions of the same images with $5 \sigma$ error circles.

SNe II-P (recently summarized by Smartt 2009). In a few cases, the candidate progenitor was confirmed by the fact that the source vanished after the SN faded; see Smartt (2009) or Leonard (2010) for detailed accounts of individual cases. Recently, there have also been some claimed detections of SN II-L progenitors which suggest progenitor stars that were somewhat more massive than those of SNe II-P (Elias-Rosa et al. 2010, 2011; see also Fraser et al. 2010; Leonard 2010). Together, the more massive progenitors of SNe II-L and IIn may require substantial modification to current views of massive-star evolution (Smith et al. 2011a).

\section{OBSERVATIONS}

The host galaxy of SN 2010jl had observations taken $\sim 10 \mathrm{yr}$ prior to discovery with the Wide Field Planetary Camera 2 (HST /WFPC2), which we retrieved from the HST archive. UGC 5189A was observed in the F300W and F814W filters on 2001 February 14 as part of GO-8645, with exposures of $1800 \mathrm{~s}$ and 200 s, respectively.

To pinpoint the precise location of the progenitor in the HST images, we obtained ground-based images of SN 2010jl for comparison using MegaCam on the $3.6 \mathrm{~m}$ Canada-France-Hawaii Telescope (CFHT). The full width at half-maximum intensity (FWHM) was 0.'6, with 0'.187 pixels. To perform astrometric solutions between the ground-based and $H S T$ images, we adopted the technique detailed by $\mathrm{Li}$ et al. (2007) using stars present in both images. Geometric transformation between a combined $600 \mathrm{~s} r$-band image (with multiple short $10 \mathrm{~s}$ exposures to ensure that SN 2010jl was not saturated) taken with MegaCam on 2010 November 9.60 and the $2001 \mathrm{HST}$ /WFPC2 images yields a precision of 0.47 WFPC2 pixels (0',047) for the SN location in the WFPC2 images. (An independent astrometric solution by one of us (S.D.V.D.) finds a larger $1 \sigma$ precision of 0.09 .) Within the positional uncertainty, an object is clearly detected in the F300W image, and marginally detected in the F814W images at $\alpha=9^{\mathrm{h}} 42^{\mathrm{m}} 53.33$, $\delta=+09^{\circ} 29^{\prime} 42^{\prime \prime} .1$ (J2000.0).

Figure 1 shows the site of SN 2010j1 in the F300W and F814W HST/WFPC2 images. A candidate progenitor source is detected within $1 \sigma$ precision of the astrometric solution. The HST photometry for the progenitor candidate as measured with
HSTphot (Dolphin 2000a, 2000b) yields F300W $=21.6 \pm$ $0.06 \mathrm{mag}$ and $\mathrm{F} 814 \mathrm{~W}=23.1 \pm 0.18 \mathrm{mag}^{7}$ The candidate $^{2}$ is surrounded by some faint extended emission and has a neighboring source within $<0$ '.4, so we forced HSTphot to recognize the position of the candidate in order to extract the photometry. Due to the complicated background, we suspect that the uncertainties of the photometry from HSTphot are underestimated, especially for the F814W filter image. The candidate source itself has FWHM less than 0 !'3, corresponding to $\sim 73 \mathrm{pc}$ at the distance of UGC 5189A.

We have also initiated a campaign to obtain intensive spectroscopy of SN 2010jl. These spectra will be analyzed in detail in a future paper, but here we briefly discuss the appearance of the early-time spectrum and the $\mathrm{H} \alpha$ profile. Figure 2 shows two spectra of SN 2010jl obtained on 2010 November 5 with the Low Resolution Imaging Spectrometer (LRIS; Oke et al. 1995) on the $10 \mathrm{~m}$ Keck I telescope, and on 2010 November 7 with the Deep Imaging Multi-Object Spectrograph (DEIMOS; Faber et al. 2003) mounted on the $10 \mathrm{~m}$ Keck II telescope. All observations were obtained with the slit oriented at the parallactic angle (Filippenko 1982). Standard routines were used to extract and calibrate the spectra.

Figure 2 compares our spectra of SN 2010jl with the earlytime (day 32) spectrum of the very luminous SN IIn 2006tf from Smith et al. (2008). Although the spectra of SNe 2010j1 and 2006tf are not identical, the continuum shape, Balmerline strengths and profiles, and presence of weak $\mathrm{He}$ I and other narrow lines indicate that the spectrum of SN 2010jl is consistent with those of SNe IIn. (There is considerable variety in the spectra of SNe IIn; see comparisons in Filippenko 1997 and Smith et al. 2010a.) The DEIMOS spectrum, which has significantly higher resolution than the LRIS spectrum, shows a number of narrow emission and absorption components from the dense pre-shock circumstellar medium (CSM). Figure 2 also illustrates a $7000 \mathrm{~K}$ blackbody for comparison, which is not a fit. The mismatch between the $7000 \mathrm{~K}$ blackbody and the

\footnotetext{
7 These transform to Vega-based magnitudes $m_{U}=22.2 \pm 0.1$ and $m_{I}=23.1 \pm 0.2$ if the source follows a $14,650 \mathrm{~K}$ blackbody for the color correction. (A blackbody of this temperature matches the apparent $m_{\mathrm{F} 300 \mathrm{~W}} / m_{\mathrm{F} 814 \mathrm{~W}}$ color, but the source may differ from a blackbody, with contributions from emission lines.)
} 


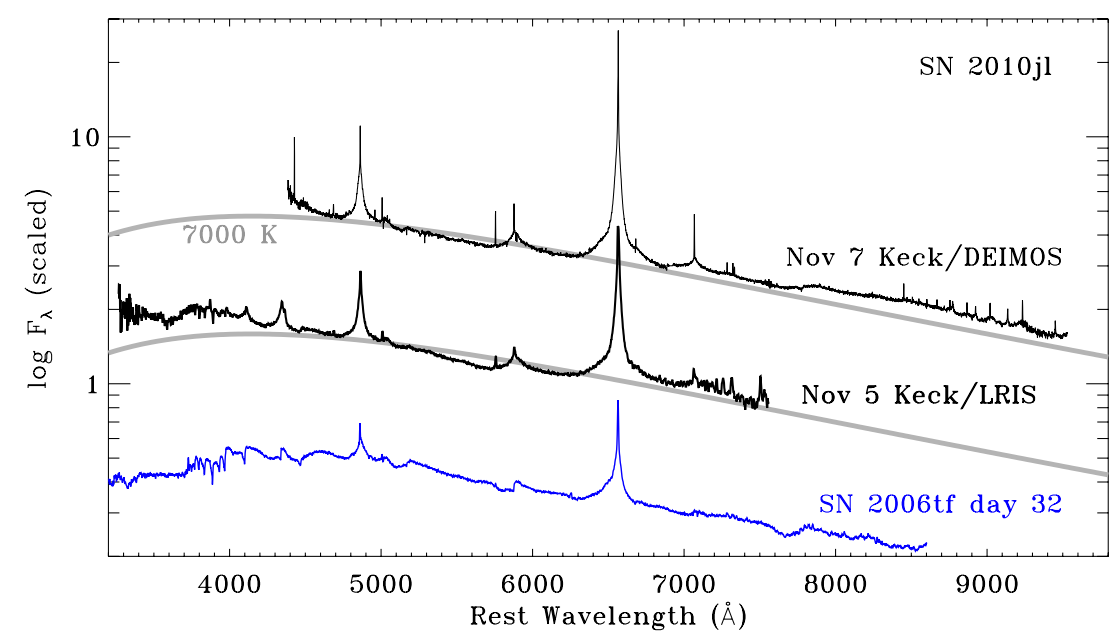

Figure 2. Optical spectra of SN 2010j1 obtained at early times on 2010 November 5 and 7 (black) compared to the day 32 spectrum of SN 2006 tf from Smith et al. (2008). All spectra are dereddened by $E(B-V)=0.027 \mathrm{mag}$ (by coincidence, SN 2006tf has the same estimated Galactic reddening value; see Smith et al. 2008). A $7000 \mathrm{~K}$ blackbody is shown in gray for comparison with the SN 2010j1 spectra (but note that a single blackbody component cannot fit the observed continuum shape corrected only for Galactic extinction).

(A color version of this figure is available in the online journal.)

observed continuum shape suggests that multiple-temperature components may be present.

Figure 3 shows the high-resolution $\mathrm{H} \alpha$ profile of SN 2010j1 observed on 2010 November 5, 6, and 7, assuming redshift $z=0.011$. Spectra on the first two nights were obtained using the Blue Channel spectrograph mounted on the Multiple Mirror Telescope (MMT), with $105 \mathrm{~s}$ exposures, a 1".0 slit width, and a spectral resolution of $\sim 4500$. The November 7 spectrum was obtained with Keck/DEIMOS, using a resolution of 4400 and a 1".0 slit. The resulting normalized spectra in Figure 3 are remarkably consistent on all three nights, despite different facilities, setups, and observing conditions. This offers reassurance that the double-peaked narrow profile is not a subtraction artifact that might arise from oversubtracting a nearby $\mathrm{H}$ II region along the slit.

The $\mathrm{H} \alpha$ profile has an intermediate-width component that can be approximated by a Lorentzian profile with FWHM = $1800 \mathrm{~km} \mathrm{~s}^{-1}$ (the thick gray curve in Figure 3), which may be common in SNe IIn at early times because of large optical depths (see Smith et al. 2010; Chugai 2001). The wings of this Lorentzian extend to more than $\pm 4000 \mathrm{~km} \mathrm{~s}^{-1}$. The Lorentzian centroid is shifted by $-50 \mathrm{~km} \mathrm{~s}^{-1}$, and the high signal-to-noise ratio spectra show some deviations from perfect symmetry in the line wings.

The narrow $\mathrm{H} \alpha$ component appears double peaked, and can be approximated by a symmetric Gaussian emission component with FWHM $=120 \mathrm{~km} \mathrm{~s}^{-1}$ (solid gray curve), but with an absorption component at $-28 \mathrm{~km} \mathrm{~s}^{-1}$ relative to the emissioncomponent centroid. The $-28 \mathrm{~km} \mathrm{~s}^{-1}$ absorption suggests that the pre-shock CSM along our line of sight is rather slow, comparable to the wind speed of an extreme red supergiant (RSG) that could be a plausible progenitor of an SN IIn (Smith et al. 2009). This is slow compared to many other SNe IIn (e.g., Kiewe et al. 2010) and to typical LBV outbursts (Smith et al. 2011b), but the scarcity of such slow CSM speeds could be an observational bias. Namely, one can only see it with relatively high-resolution spectra (the narrow absorption component is unresolved in our lower-resolution Keck/LRIS spectrum, for example), while faster absorption components may be easier to detect in the low-resolution spectra that are more commonly obtained. However, LBV outflows can

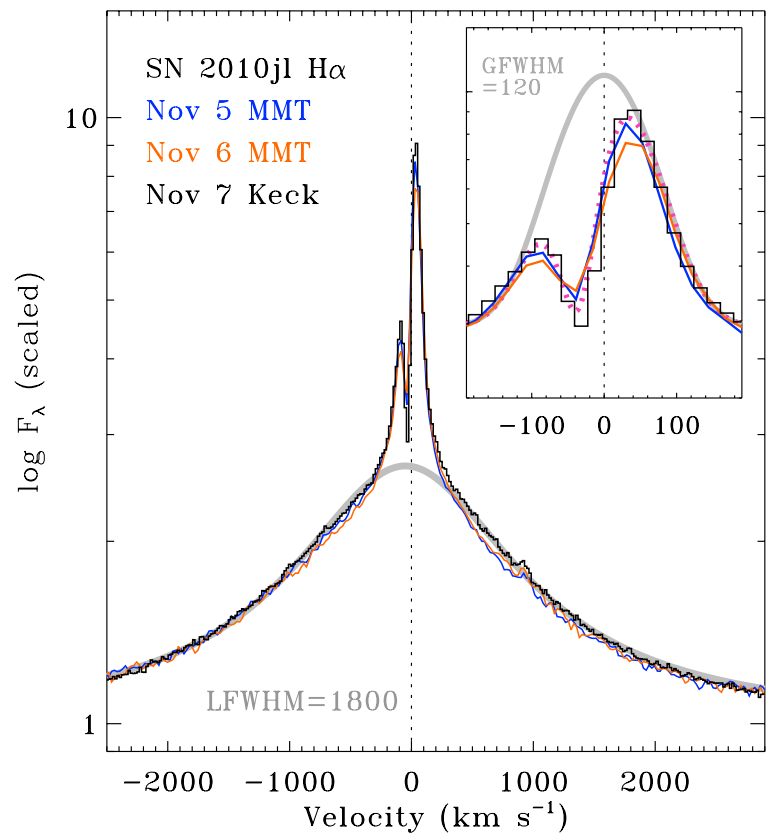

Figure 3. H $\alpha$ profile of SN 2010jl on 2010 November 5 (blue), 6 (orange), and 7 (black histogram), taken with the MMT Blue Channel spectrograph and Keck/ DEIMOS. These correspond to days 2, 3, and 4 after discovery, respectively, and show little change with time or observing parameters (see the text). The thick gray curve is a Lorentzian profile with FWHM $=1800 \mathrm{~km} \mathrm{~s}^{-1}$. The inset shows the narrow profile on an expanded velocity scale. The gray curve here is a symmetric Gaussian with FWHM $=120 \mathrm{~km} \mathrm{~s}^{-1}$, while the dotted magenta curve is the same, but with a narrower blueshifted Gaussian subtracted (centered at $-28 \mathrm{~km} \mathrm{~s}^{-1}$, FWHM $=64 \mathrm{~km} \mathrm{~s}^{-1}$ ).

(A color version of this figure is available in the online journal.)

be highly asymmetric - the nebula around $\eta$ Car has outflow speeds of $\sim 40 \mathrm{~km} \mathrm{~s}^{-1}$ at low latitudes, and higher speeds up to $650 \mathrm{~km} \mathrm{~s}^{-1}$ at the poles (Smith 2006). Thus, the $120 \mathrm{~km} \mathrm{~s}^{-1}$ emission component could be due to faster, ionized, pre-shock wind in directions away from our line of sight, while we may see slower speeds in absorption if we are viewing SN 2010jl from low latitudes. Alternatively, at such early times (and relatively small radii in the CSM), radiative acceleration of the preshock CSM by the SN light may also play a role (e.g., Chugai 


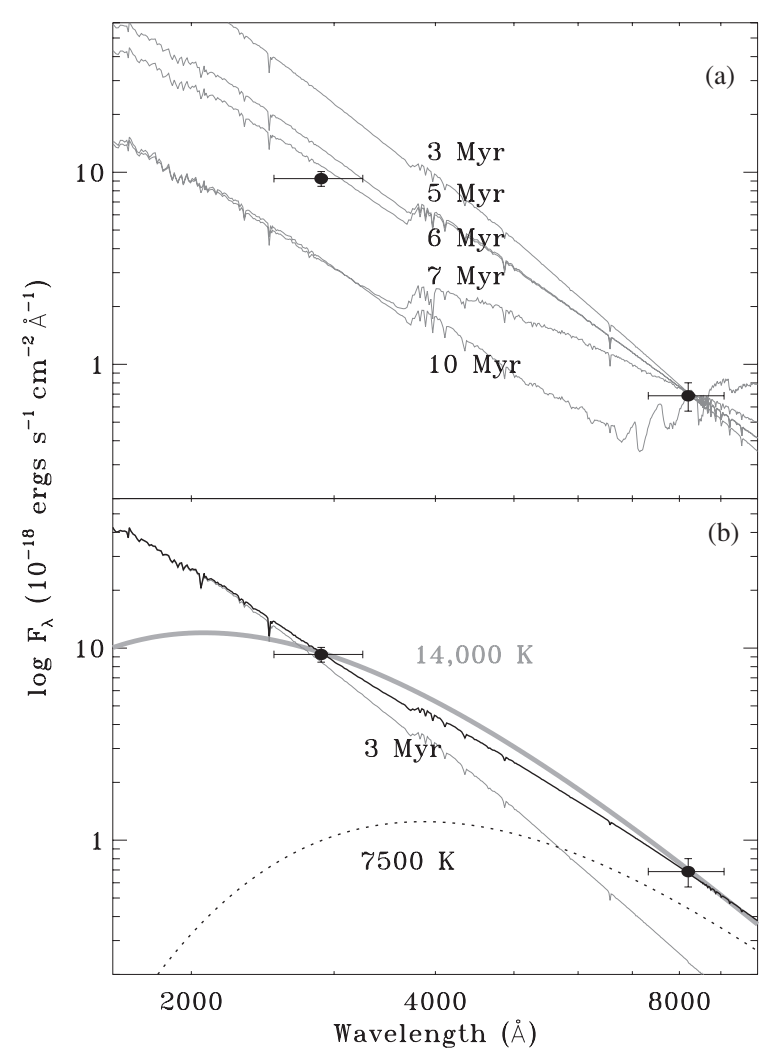

Figure 4. Black points in both panels are the fluxes of the candidate progenitor derived from the magnitudes measured in F300W and F814W WFPC2 images, dereddened by $E(B-V)=0.027$ mag as described in the text. Panel (a) compares this photometry to Starburst99 (Leitherer et al. 1999) models of the integrated spectrum of a massive star cluster with ages of 3, 5, 6, 7, and $10 \mathrm{Myr}$. Panel (b) shows the same photometry, but compared to a 14,000 K blackbody (thick gray line), and a composite spectrum (thin black line) that results from the combination of a 3 Myr cluster (same as above) and a $7500 \mathrm{~K}$ blackbody (dotted line), as might be expected from a cool LBV.

et al. 2002), although in this case it would be unclear why the $28 \mathrm{~km} \mathrm{~s}^{-1}$ component along our line of sight is not accelerated.

\section{LIKELY INTERPRETATIONS}

We adopt a distance to UGC 5189A of $48.9 \pm 3.4 \mathrm{Mpc}$ (distance modulus $m-M=33.45 \pm 0.15 \mathrm{mag})^{8}$ and a Galactic reddening value of $E(B-V)=0.027 \mathrm{mag}\left(A_{U}=0.149 \mathrm{mag}\right.$, $A_{I}=0.053 \mathrm{mag}$ ) from Schlegel et al. (1998). HST magnitudes were converted to flux using the zero-magnitude fluxes given in Table 9 of Holtzman et al. (1995). We do not assume any host-galaxy reddening in our analysis below, since the $\mathrm{Na}$ I D1 equivalent width is only about $0.12 \AA$. With these parameters, the apparent magnitudes imply a very luminous source with absolute magnitudes of about -12.0 (F300W) and -10.4 (F814W). Possible interpretations of this luminous blue source are as follows.

1. The SN progenitor resided in a blue star cluster. If the blue source detected in the HST images is not dominated by emission from the progenitor star itself, it could be a luminous blue star cluster at the same position, of which the progenitor may have been a member. Figure 4(a) shows that the blue color of the source could be explained by a young star cluster with an age of 5-6 Myr. If the progenitor candidate of SN 2010jl is actually a young

\footnotetext{
8 This distance is from the NASA/IPAC Extragalactic Database: http://nedwww.ipac.caltech.edu/
}

blue star cluster, it is among the most massive young star clusters known. Even in colliding starburst galaxies like the Antennae, clusters with $M_{V}<-10$ mag are extremely rare (Whitmore et al. 2010). As a more familiar example in a dwarf irregular galaxy, the entire 30 Doradus complex has an absolute visual magnitude of about -11 , but this would be spread over $\sim 1^{\prime \prime} .5$ at the distance of UGC $5189 \mathrm{~A}$. The more compact star cluster R136 in the core of 30 Dor has an absolute magnitude of only about $-9.3 \mathrm{mag}$, and would be spatially unresolved in UGC 5189A. It is probable that any member of such a young star cluster reaching core collapse would be among the most massive stars in that cluster, and a cluster age of $<7$ Myr implies a stellar lifetime corresponding to initial masses $>30 M_{\odot}$ (e.g., Schaller et al. 1992), if the cluster is roughly coeval to within about $1 \mathrm{Myr}$.

2. The SN progenitor was an extremely luminous $L B V$-like star in quiescence. If an absolute F814W magnitude of -10.4 corresponds to an individual star, that star was extremely luminous and massive. The most massive main-sequence O-type stars do not have visual luminosities this high, because they are too hot and they emit most of their flux in the ultraviolet. To be this bright at red wavelengths, a star would need to be evolved, shifting its bolometric flux to longer wavelengths. However, even the most massive yellow hypergiants and RSGs have bolometric luminosities fainter than about -9.5 mag (Humphreys \& Davidson 1979), and they are redder than the progenitor candidate, so these sources cannot account for the detected object. The dereddened color is consistent with an apparent temperature of roughly 14,000 K (Figure 4(b)). The only viable type of quiescent blue star would be an extremely luminous LBVlike star, but it would need a luminosity comparable to the most luminous known stars such as $\eta$ Car, implying an initial mass above $80 M_{\odot}$. This is similar to the case for the progenitor of SN 2005gl (Gal-Yam et al. 2007).

3. The SN progenitor was normally fainter, but was caught in a precursor $L B V$-like eruption phase. One could relax the requirement that the progenitor of $\mathrm{SN} 2010 \mathrm{jl}$ was among the most massive stars known if the star was in an outburst state at the time it was observed by HST. An absolute F814W magnitude of -10.4 with a blue color is within the range of observed values for LBV-like eruptions, either as a bright $S$ Doradus eruption or a relatively modest example of a giant LBV eruption (see Smith et al. 2011b for details). The blue color, though, would be more consistent with the latter (Smith et al. 2011b). This explanation has the advantage that a precursor LBV-like eruption is needed anyway, in order to create the dense CSM needed to explain the Type IIn spectrum and high luminosity of the SN (e.g., Smith et al. 2008). Since these outbursts can, in some cases, last for $\sim 10$ yr (see Smith et al. 2011b), it is not necessarily improbable to catch a progenitor star in this phase within the decade before core collapse.

4. A combination of the above. It is also possible that the detected flux from the progenitor candidate has contributions from both a host cluster and option (2) or (3) above. However, as shown in Figure 4(b), if most of the red flux comes from a cool LBV with an apparent temperature around 7500 $\mathrm{K}$, for example, then this tightens the restrictions on the cluster age: the cluster must be bluer and therefore younger than for a cluster alone, implying an age of 3 Myr or less. By the same line of reasoning as discussed above, this younger age would imply an even more massive progenitor. 
Hypothetically, there is a very small probability that an unrelated star cluster would be seen at the $\mathrm{SN}$ position due to a chance line-of-sight projection. For a $20 \times 20$ pixel area around the SN location $\left(2^{\prime \prime} .0 \times 2\right.$ ". 0$), 13$ sources were detected in the F300W image at the $3 \sigma$ level. For an error radius of 0.47 pixel, the chance for a random coincidence is only $2.3 \%$. A chance projection is therefore very unlikely; moreover, this type of ambiguity plagues all studies of SN host sites. To confirm that our candidate source detected in archival data was in fact the direct detection of a luminous progenitor star will require additional observations after the SN has faded, to see if it has significantly changed-but for an SN IIn that may continue to interact with dense CSM, we may need to wait several years. Even before that time, however, one significant point is clearly evident: all plausible scenarios require the progenitor of SN 2010jl to have been a very massive star, with an initial mass higher than those typically derived for SNe II-P (Smartt 2009; Leonard 2010). This has significant implications for stellar evolution.

\section{IMPLICATIONS FOR MASSIVE-STAR EVOLUTION}

Whether the progenitor candidate is a young star cluster or a direct detection of the progenitor star itself, the luminous blue source implies that the progenitor had an initial mass above $30 M_{\odot}$. SN progenitors below this range, as seen for SNe II$\mathrm{P}$ (Smartt 2009), are not found to reside in very luminous, compact, young star clusters, although $\sim 10 \%$ of SNe II-P are associated with older star clusters that remain bound. An individual star with a quiescent luminosity of the candidate progenitor would have an initial mass $\gtrsim 80 M_{\odot}$, and a star caught in an LBV outburst would most likely be a star with an initial mass above $30 M_{\odot}$ as well (see Smith et al. 2011b).

A massive-star progenitor for SN 2010jl adds to mounting evidence for three general conclusions concerning the fates of massive stars, as follows.

1. SNe IIn arise preferentially from very massive stellar progenitors. As noted in Section 1, this is based on the direct detections of LBV-like progenitors of SN 2005gl and SN 1961V (Gal-Yam et al. 2007; Gal-Yam \& Leonard 2009; Smith et al. 2011b; Kochanek et al. 2010; but see Van Dyk \& Matheson 2011), as well as on the large amounts of mass in the CSM needed to explain luminous SNe IIn (e.g., Smith et al. 2007). If the SN 2010jl progenitor candidate is a luminous individual star resembling an LBV, it further strengthens the connection between LBVs and SNe IIn.

2. Since SN 2010jl is an SN IIn, requiring that the progenitor ejected H-rich material shortly before core collapse, its massive progenitor reinforces the conclusion that very massive stars can sometimes retain a hydrogen envelope until shortly before core collapse (Smith \& Owocki 2006), instead of shedding all of their $\mathrm{H}$ envelope at nearly solar metallicity to produce SNe Ibc (e.g., Heger et al. 2003). A viable alternative, which is consistent with the observed fractions of various $\mathrm{SN}$ subtypes, is that many $\mathrm{SNe} \mathrm{Ibc}$ result instead from close binary evolution across a wide range of progenitor mass, and that the most massive single stars produce SNe IIn (Smith et al. 2011a; Yoon et al. 2010). Alternatively, Gal-Yam et al. (2007) proposed that stars of initial mass $80-150 M_{\odot}$ explode as LBVs to make SNe IIn, whereas stars of 40-80 $M_{\odot}$ explode as SNe Ic.

3. Lastly, if the SN 2010jl progenitor was a massive star, it provides another example suggesting that very massive stars can produce luminous explosions, instead of collapsing qui- etly to a black hole (see O'Connor \& Ott 2011; Smith et al. 2011a; Kochanek et al. 2008).

Standard stellar evolution models fail to account for all three of these basic observational indications.

The work presented here is based in part on observations made with the NASA/ESA Hubble Space Telescope, obtained at the Space Telescope Science Institute, which is operated by the Association of Universities for Research in Astronomy, Inc., under NASA contract NAS5-26555; the MMT Observatory, a joint facility of the Smithsonian Institution and the University of Arizona; and MegaPrime/MegaCam, a joint project of Canada-France-Hawaii Telescope (CFHT) and CEA/DAPNIA, at the CFHT which is operated by the National Research Council (NRC) of Canada, the Institut National des Science de l'Univers of the Centre National de la Recherche Scientifique (CNRS) of France, and the University of Hawaii. Some of the data presented herein were obtained at the W. M. Keck Observatory, which is operated as a scientific partnership among the California Institute of Technology, the University of California, and NASA; the observatory was made possible by the generous financial support of the W. M. Keck Foundation. We thank the staffs at these observatories for their efficient assistance, as well as R. J. Foley and S. B. Cenko for their help at Keck. Supernova research of A.V.F.'s group at U. C. Berkeley is supported by National Science Foundation grant AST-0908886, by the TABASGO Foundation, and by NASA through grants AR-11248 and AR-12126 from the Space Telescope Science Institute, which is operated by Associated Universities for Research in Astronomy, Inc., under NASA contract NAS 5-26555. J.M.S. is grateful to Marc J. Staley for a Graduate Fellowship. We thank J. R. Graham and S. Wright for obtaining Keck adaptive optics images of the site of SN 2010jl for us; unfortunately, these data did not yield a useful astrometric solution because only the SN was clearly detected.

Facilities: HST(WFPC2), Keck:I(LRIS), Keck:II (DEIMOS), MMT (Blue Channel), CFHT (MagaCam)

\section{REFERENCES}

Benetti, S., Bufano, F., Vinko, J., Marion, G. H., Pritchard, T., \& Wheeler, J. C. 2010, CBET, 2536, 1

Chugai, N. N. 2001, MNRAS, 326, 1448

Chugai, N. N., Blinnikov, S. I., Fassia, A., Lundqvist, P., Meikle, W. P. S., \& Sorokina, E. I. 2002, MNRAS, 330, 473

Dolphin, A. E. 2000a, PASP, 112, 1383

Dolphin, A. E. 2000b, PASP, 112, 1397

Elias-Rosa, N., et al. 2010, ApJ, 714, L254

Elias-Rosa, N., et al. 2011, submitted

Faber, S. M., et al. 2003, Proc. SPIE, 4841, 1657

Filippenko, A. V. 1982, PASP, 94, 715

Filippenko, A. V. 1997, ARA\&A, 35, 309

Filippenko, A. V., Barth, A. J., Bower, G. C., Ho, L. C., Stringfellow, G. S., Goodrich, R. W., \& Porter, A. C. 1995, AJ, 110, 2261

Fraser, M., et al. 2010, ApJ, 714, L280

Gal-Yam, A., \& Leonard, D. C. 2009, Nature, 458, 865

Gal-Yam, A., et al. 2007, ApJ, 656, 372

Goodrich, R. W., Stringfellow, G. S., Penrod, G. D., \& Filippenko, A. V. 1989, ApJ, 342, 908

Heger, A., Fryer, C. L., Woosley, S. E., Langer, N., \& Hartmann, D. H. 2003, ApJ, 591,288

Holtzman, J. A., Burrows, C. J., Casertano, S., Hester, J. J., Trauger, J. T., Watson, A. M., \& Worthey, G. 1995, PASP, 107, 1065

Humphreys, R. M., \& Davidson, K. 1979, ApJ, 232, 409

Kiewe, M., et al. 2010, arXiv:1010.2689

Kochanek, C. S., Beacom, J. F., Kistler, M. D., Prieto, J. L., Stanek, K. Z., Thompson, T. A., \& Yüksel, H. 2008, ApJ, 684, 1336

Kochanek, C., et al. 2010, arXiv:1010.3704 
Leitherer, C., et al. 1999, ApJS, 123, 3

Leonard, D. C. 2010, Ap\&SS, online, doi 10.1007/s10509-010-5380-8

Li, W., et al. 2007, ApJ, 661, 1013

Li, W., et al. 2011, MNRAS, in press (arXiv:1006.4612)

Newton, J., \& Puckett, T. 2010, CBET, 2532, 1

O'Connor, E., \& Ott, C. D. 2011, ApJ, 730, 70

Oke, J. B., et al. 1995, PASP, 107, 375

Schaller, G., Schaerer, D., Meynet, G., \& Maeder, A. 1992, A\&AS, 96, 269

Schlegel, D. J., Finkbeiner, D. P., \& Davis, M. 1998, ApJ, 500, 525

Schlegel, E. 1990, MNRAS, 244, 269

Smartt, S. J. 2009, ARA\&A, 47, 63

Smith, N. 2006, ApJ, 644, 1151

Smith, N. 2008, in IAU Symp. 250, Massive Stars as Cosmic Engines, ed. F. Bresolin, P. A. Crowther, \& J. Puls (Cambridge: Cambridge Univ. Press), 193

Smith, N., Hinkle, K. H., \& Ryde, N. 2009, AJ, 137, 3558
Smith, N., Li, W., Filippenko, A. V., \& Chornock, R. 2011a, MNRAS, in press (arXiv:1006.3899)

Smith, N., Li, W., Silverman, J. M., Ganeshalingam, M., \& Filippenko, A. V. 2011b, MNRAS, in press (arXiv:1010.3718)

Smith, N., \& McCray, R. 2007, ApJ, 671, L17

Smith, N., \& Owocki, S. P. 2006, ApJ, 645, L45

Smith, N., et al. 2007, ApJ, 666, 1116

Smith, N., et al. 2008, ApJ, 686, 467

Smith, N., et al. 2010, ApJ, 709, 856

Van Dyk, S. D. 2005, The Fate of the Most Massive Stars, ASP Conf. Ser. 332 (San Francisco: ASP), p. 47

Van Dyk, S. D., \& Matheson, T. 2011, ApJ, submitted

van Marle, A. J., Smith, N., Owocki, S. P., \& van Veelen, B. 2010, MNRAS, 407, 2305

Whitmore, B. C., et al. 2010, AJ, 140, 75

Yoon, S. C., Woosley, S. E., \& Langer, N. 2010, ApJ, 725, 940 